\title{
Autoimmune disorders in child psychiatry: keeping up with the field
}

\author{
Adele Warrilow \& Michael Morton
}

\begin{abstract}
SUMMARY
Autoimmune disorders in children and adolescents can have significant neuropsychiatric complications and there is growing interest in the association between autoimmune conditions and psychiatric syndromes, particularly in Down syndrome. Acute presentations with psychiatric symptoms require careful assessment in order to recognise and plan treatment of underlying autoimmune disease in collaboration with paediatric colleagues. Difficult treatment decisions arise in children with established autoimmune diagnoses and psychiatric symptoms that may be a result of neuroimmunological processes associated with their condition, psychiatric side-effects of drug treatments or psychopathology resulting from other factors in the history that may or may not have a direct relation to the autoimmune diagnosis. This article illustrates these complexities through discussion of specific autoimmune disorders and three case histories.
\end{abstract}

\section{LEARNING OBJECTIVES}

- Be aware of the impact of a rapidly changing knowledge base in autoimmune disorders across a wide range of areas of practice in child and adolescent psychiatry

- Know when to suspect autoimmune disorder and how to pursue a diagnosis

- Know how to evaluate and respond to requests for advice from paediatric colleagues concerned about children with autoimmune disorders

\section{DECLARATION OF INTEREST}

None

There has been a rapid development of knowledge of the role of autoimmunity in common medical conditions and also in psychiatric presentations and intellectual disability. Autoimmune disorders form a heterogeneous group of conditions mediated by a variety of mechanisms, which in turn are modulated by gene and environment interactions that are not yet fully understood. Various tried and tested medical therapeutic approaches are widely used in a range of clinical settings, but they are rarely considered in relation to psychiatric presentations. Although autoimmune conditions were previously considered to be entirely discrete disorders, it is now recognised that there is a significant rate of co-occurrence of multiple different autoimmune disorders at both the individual and family level. For example, in Down syndrome an increased risk of a number of autoimmune disorders reflects a range of defects in the immune system associated with the genotype (Box 1) (Chistiakov 2007).

It has been estimated that it takes an average of 17 years for new scientific discoveries to be translated into routine clinical practice (Morris 2011). The realisation that the blood-brain barrier (made particularly vulnerable by stress, infections and inflammation) is variably permeable to autoimmune complexes has been a significant advance in understanding the brain effects of autoimmune disease. Postulated mechanisms include autoimmune effects on neurotransmitter production, ion channels and the integrity and functioning of neurons and microglia, mediated by autoantibodies (many not yet identified) and the complement system. The role of immunology in normal brain development is also

BOX 1 Autoimmune disease in Down syndrome

A triad of autoimmune disorders is commonly found in association with Down syndrome:

- type 1 diabetes

- Hashimoto's disease

- coeliac disease

Suggested monitoring of children with Down syndrome to be reviewed in any presentation to child and adolescent mental health services:

- monitor growth

- screen for signs and symptoms of diabetes and thyroid disease

- monitor thyroid function tests (note: annual thyroid function tests are advised in all individuals with Down syndrome), screen for anti-thyroid and anti-islet cell antibodies, measure serum levels of antigliadin and anti-endomysium autoantibodies

(Chistiakov 2007)
Adele Warrilow is a PSYSTAR Clinical Research Fellow at the Institute of Health and Wellbeing, University of Glasgow and a higher specialty trainee in child and adolescent psychiatry in the West of Scotland. Michael Morton is honorary clinical senior lecturer in child and adolescent psychiatry in the Institute of Health and Wellbeing, University of

Glasgow. He retired from the post of consultant child and adolescent psychiatrist with a special interest in neuropsychiatry at the Royal Hospital for Sick Children, Glasgow in December 2014. Correspondence Dr Michael Morton, Institute of Health \& Wellbeing, College of Medical, Veterinary and Life Sciences, University of Glasgow, Caledonia House, Royal Hospital for Sick Children, Yorkhill, Glasgow G3 8SJ, UK. Email: Michael.morton@ glasgow.ac.uk 
a rapidly emerging area of research, for example microglia have a function in relation to synaptic connection that has an impact on neural circuitry (Leckman 2015).

The potential impact of improved diagnosis and treatment of autoimmune disorders on psychiatric practice makes this an exciting area for psychiatrists and clinical researchers to keep abreast of and they have a responsibility to ensure that patients benefit as soon as possible. A recent editorial in the journal Brain Research concludes cautiously that 'while work in developmental psychoneuroimmunology engenders a good deal of excitement, the "promise" of the field clearly remains greater than the "deliverables", in terms of any direct benefits on patient care' (Leckman 2015). Considering the need to find a balance between the future possibilities implied by current research and the realities of clinical practice, this article aims to give a practical summary of autoimmune disorders in child psychiatry, concentrating on the 'deliverables' within current practice - recognition and a multidisciplinary liaison approach to assessment and therapy.

\section{Presentation to psychiatric services}

Children with autoimmune disorders presenting to psychiatry can be thought of in three main ways:

- those with neuropsychiatric complications of a known autoimmune disorder (paediatricians may ask for psychiatric input in case management)

- those with psychiatric disorder and a co-occurring autoimmune disorder (collaboration of paediatric and mental health teams could be helpful)

- those with what appears to be a primarily psychiatric illness but with indicators suggesting autoimmune aetiology (where the child and adolescent psychiatrist may need to seek the help of a paediatrician).

This categorisation, although helpful, is an oversimplification as disease processes can occur separately or simultaneously and it may not be possible in current clinical practice accurately to apportion presenting psychiatric symptoms to a single particular aetiology or mechanism. As in all medical specialties, any presentation is in the context of individual and family biological, psychological and social characteristics. Given the range of autoimmune disorders and the variation in the current levels of scientific understanding of the interaction of psychiatric and medical conditions, this article will illustrate key points that may apply across various disorders, by discussion of a selection of specific clinical presentations, some common and some extremely rare.

\section{Common autoimmune medical conditions}

\section{Psoriasis}

Psoriasis is an autoimmune skin condition with, as yet, no known direct cerebral autoimmune involvement. However, it is important to note that recently, psoriasis has been recognised as being more of a systemic illness than traditionally thought (Laws 2014). Psoriasis has a significant negative impact on quality of life, well-being and social functioning. Living with a chronic condition that causes itchy, flaking skin and can also affect nails and joints can be difficult. The visibility of the illness can result in lowered selfesteem, social stigma and bullying, and in some circumstances a vicious cycle of stress leading to worsening of the condition can be identified. The treatment of psoriasis may have an effect on mental health, for example large areas treated with potent corticosteroids can result in systemic effects, and mood changes are a recognised sideeffect of methotrexate therapy. In some cases, of course, the presenting psychiatric illness is entirely unrelated to the diagnosis of psoriasis, and autoimmune disorder is a coincidental finding in the patient's history.

\section{Type 1 diabetes}

In child and adolescent type 1 diabetes, mental health problems occur with greater frequency than microvascular and other comorbid autoimmune conditions (Cameron 2012). Possibly most well recognised are the psychological difficulties of diabetes as a chronic disease occurring alongside childhood and adolescent psychological and social development, including the transition to self-management of parenteral insulin. The magnification of self-harm risks associated with insulin use increases the challenge in managing comorbid psychiatric disorder. The metabolic effects of hypoglycaemia and hyperglycaemia can also affect the brain. For example, children who have an initial presentation of diabetic ketoacidosis are more likely to have morphological brain changes and difficulties with memory and concentration at least in the medium term (Cameron 2014). There are also potentially direct brain effects of the disease process. Type 1 diabetes is associated with autoantibodies against pancreatic glutamic acid decarboxylase (GAD), which may also show affinity for brain-expressed GAD, a key enzyme in the synthesis of the neurotransmitter gammaaminobutyric acid (GABA) from glutamate (Benros 2014). Type 1 diabetes has increased prevalence in Down syndrome and its diagnosis may be delayed if this risk is not borne in mind. 


\section{Hashimoto's disease}

Hashimoto's disease is characterised by an autoimmune thyroiditis that is the most common cause of thyroid disease in children, with prevalence rates of up to $3 \%$ in some populations (Sari 2011). This disorder has a genetic basis and may be triggered by infection. Damage to thyroid tissue is mediated by T-lymphocyte production of thyroidspecific autoantibodies. The insidious onset of hypothyroidism, with symptoms that may be suggestive of hyperthyroidism (such as excessive sweating, nervousness, irritability and overactivity) can lead to delayed diagnosis, particularly as goitre is not necessarily present. More rarely, psychotic symptoms associated with gradual cognitive decline can point to the development of Hashimoto's encephalopathy, which is a rare condition with a diagnosis confirmed by the presence of elevated thyroid autoantibodies (Sari 2011). In Down syndrome a clinical diagnosis of thyroid disorder may be difficult, but there is a high prevalence of Hashimoto's disease, with a risk that reversible cognitive decline and behavioural disturbance may be missed or even misdiagnosed as an Alzheimer-type presentation (which is recognised as a risk in young adults with Down syndrome).

\section{Coeliac disease}

An association between coeliac disease and psychiatric illness has been hypothesised for many years - observational studies in the 1950s and 1960s described a higher than usual number of patients with both schizophrenia and coeliac disease. More recent population studies have been inconclusive (Benros 2014). Similarly, there has been controversy about a possible link between coeliac disease and autism spectrum disorder (ASD); this raised the possibility of a false association, as children with ASD might be more likely to be screened for coeliac disease as a result of the debate. A recent Swedish nationwide study failed to confirm this link, but did provide evidence suggesting increased 'non-coeliac gluten intolerance' in individuals with ASD (Ludvigsson 2013). The classification of coeliac disease and gluten sensitivity is an evolving area. The extraintestinal manifestations of coeliac disease (previously called atypical symptoms) are now recognised as being more common than was thought. As with other gastrointestinal disorders, abdominal symptoms are not always prominent and children with coeliac disease may present with symptoms including irritability, chronic fatigue, anorexia and weight loss or failure to thrive (Box 2) (Tonutti 2014). Coeliac disease is
BOX 2 Case vignette: anorexia and coeliac disease

An 11-year-old girl presented to child and adolescent mental health services with dramatic weight loss associated with dietary restriction, low mood, fatigue and a morbid fear of fatness. Her extreme presentation led to an early psychiatric admission. Serological screening followed by an intestinal biopsy showing gluten enteropathy led to a trial of a gluten-free diet. Rapid weight gain on a suitable diet was accompanied by an improvement in mental state to the point that community management of her eating disorder was achieved swiftly. Understanding the role of her physical condition in the formulation was central to management of her illness.

the third of a triad of conditions with increased prevalence in Down syndrome (Box 1). This is important as there may be a reversible impact of malabsorption on growth and development that might be misattributed to the underlying chromosomal abnormality.

\section{Rheumatoid arthritis}

The changing understanding of rheumatoid arthritis illustrates the advances in medical knowledge over the past few years. It is not long ago that rheumatoid arthritis was considered to be a classic psychosomatic illness, with a poor maternal relationship in childhood a hypothesised risk factor (Baker 1981). The possible impact of early life stress on the functioning of the hypothalamic-pituitary-adrenal (HPA) axis in an inflammatory disorder is now better understood (Essex 2011), but the confident connection of stress with the progression of rheumatoid arthritis is no longer made. The psychosocial burden on families associated with a child's diagnosis of rheumatoid arthritis remains an important consideration for paediatric services. In addition, as with other severe autoimmune conditions, consideration must be given to the neuropsychiatric side-effects of medication and also to the effects on the brain of systemic inflammation, including altered brain metabolism, recognised as occurring even in the absence of direct autoimmune attack on the brain (Emmer 2009).

\section{Systemic lupus erythematosus}

Systemic lupus erythematosus (SLE) can present with a variety of symptoms affecting almost any physiological system, for example the characteristic butterfly rash, gastrointestinal disturbance, pulmonary disorders and renal disease, in addition to signs of systemic illness such as fatigue or unexplained fever. The 
American College of Rheumatology (1999) has described 19 neuropsychiatric syndromes of SLE, including: acute confusional state, anxiety disorder, cognitive dysfunction, mood disorder and psychosis, as well as chorea and seizure disorders. Paediatric SLE is associated with a high prevalence of neuropsychiatric complications, with approximately half of children with SLE experiencing at least one neuropsychiatric symptom (Chan 2000). SLE should be held in mind as an important differential diagnosis in young people presenting with an acute onset of psychiatric symptoms, particularly if the presentation is atypical, if there are associated physical symptoms or signs or a strong family history of autoimmune disorder. When psychiatric disorder arises in association with an established diagnosis of SLE, consideration should be given in the formulation to the possible impact of neuropsychological changes, but care should be taken that the potential neuropsychiatric impact of SLE is not overemphasised, leading to neglect of other factors that may be independent of the disease process and open to change.

\section{Neuropsychiatric disorders}

In susceptible individuals, certain infections can trigger the onset of autoimmune disorders, resulting in further inflammation. Neuropsychiatric complications may arise from the inflammation associated with the infection and from autoimmune disease, which can affect the brain both independently and synergistically (Benros 2013). Swedo et al (2012) have developed the concept of paediatric acute-onset neuropsychiatric syndrome (PANS) as a way of describing a range of presentations.

\section{Post-streptococcal disorders}

Sydenham's chorea

Sydenham's chorea is the major neurological presentation of rheumatic fever, an inflammatory process following infection with group A streptococci (GAS) such as throat infection or scarlet fever. There is evidence that family genetic predisposition may be important in some cases. Sydenham's chorea is characterised by uncoordinated jerking movements affecting the face and limbs and hypotonia. Some children also experience vocal and motor tics that may persist (Box 3). Behavioural symptoms and psychiatric comorbidities are also common and can have an abrupt onset which can precede the onset of the chorea. Emotional lability, anxiety and symptoms of obsessive-compulsive disorder (OCD) are the
BOX 3 Case vignette: tic disorder after Sydenham's chorea

A boy of 8 presented with an acute-onset movement disorder. As the presentation was suggestive of Sydenham's chorea, he was referred to the paediatric neurosciences service. At the specialist clinic several weeks later, the child had no chorea but had an obvious motor and vocal tic disorder. He was able to say that 'his movements' had changed. Review of a family video made earlier in the illness confirmed a diagnosis of Sydenham's chorea, leading to discovery of cardiac involvement and a prescription for long-term penicillin. The child continued to tic and fulfilled criteria for a diagnosis of Tourette syndrome, with symptoms worsening during or after childhood illnesses. Understanding the impact of immune factors helped to guide treatment, clonidine was helpful and a tonsillectomy reduced the frequency of relapses.

most well recognised and there have also been associations with attention-deficit hyperactivity disorder (ADHD), mood disorders and even symptoms of psychosis. All of these conditions can have an impact on learning and development, particularly if they are not identified. In addition, it is possible that impairments in executive functioning may be associated with Sydenham's chorea in the absence of a psychiatric diagnosis. Sydenham's chorea usually resolves spontaneously within a few months, although it can occasionally lead to persistent symptoms of chorea and/or fatigue. It may become a recurring illness, with or without further infection; pregnancy and the oral contraceptive pill may precipitate relapses of chorea. The recognised complications of Sydenham's chorea include joint pains and damage to heart valves, as well as psychiatric symptoms. Basal ganglia damage and impaired executive functioning have been shown to persist into adulthood in some cases (Williams 2015).

Paediatric autoimmune neuropsychiatric disorder associated with streptococcal infections (PANDAS)

Paediatric autoimmune neuropsychiatric disorder associated with streptococcal infections (PANDAS) describes a disorder within the PANS classification characterised by sudden and dramatic onset of OCD, tic symptoms or severely restricted food intake and neuropsychiatric comorbidity, also of sudden and severe onset, following GAS infection. The timescale for onset of symptoms after infection is variable. Acute rheumatic fever, Sydenham's chorea and other medical disorders should be excluded. Categories of comorbidity are similar to those of Sydenham's chorea and include: anxiety (including separation anxiety); emotional lability 
BOX 4 Case vignette: anti-NMDA receptor encephalitis

A 13-year-old boy attended the emergency department, hallucinating and disoriented after an epileptic seizure. There was a history of several weeks of increasing disciplinary problems and declining school performance. Drug screen was negative. Following admission, he had poor sleep and episodes of aggression and confusion, his temperature, pulse and blood pressure were fluctuating and he developed orofacial dyskinesia. Combined assessment by a child neurologist and child psychiatrist achieved early diagnosis of anti-NMDA receptor encephalitis with raised antibodies, and initiation of immunotherapy. Physical recovery was good, but persistent memory impairment complicated his return to school. He required long-term psychological support to re-engage with education.

and/or depression; irritability, aggression and/ or severely oppositional behaviours; behavioural (developmental) regression; deterioration in school performance (ADHD-like symptoms, memory deficits, cognitive changes); sensory or motor abnormalities; somatic signs and symptoms, including sleep disturbances, enuresis or increased urinary frequency (Swedo 2012). These symptoms can have a significant impact on quality of life. A recent study (Murphy 2015) found that nearly a third of children assessed endorsed items related to suicide on the Child Behavior Checklist.

\section{Encephalitides}

Children with encephalitis may present to psychiatry with a variety of symptoms dependent on the subtype. Although many of these presentations are thought to have a post-infection origin some may be paraneoplastic (i.e. associated with underlying cancerous conditions).

\section{Limbic encephalitis}

Limbic encephalitis is characterised by an acute onset of memory loss, confusion and seizures and has traditionally been considered either paraneoplastic or viral in origin. However, it has been increasingly recognised that autoimmune forms of limbic encephalitis can occur in the absence of infection or malignancy. The term limbic encephalitis relates to inflammation of the limbic system, including the medial temporal lobes, amygdala and cingulate gyrus, but the label has historically often been applied erroneously to any encephalopathy with seizures, and memory and behavioural difficulties (Armangue 2012). Limbic encephalitis is rare in children and is associated with significant pathology.
The most frequent paediatric autoimmune encephalitis diagnosis is anti- $N$-methyl-D-aspartate (anti-NMDA) receptor encephalitis with potassium channel dysfunction mediated by antibodies targeting the NR1 receptor subunit (Dalmau 2008) (Box 4). In adults and teenagers, the illness usually follows a staged course (Fig. 1), beginning with a prodromal stage of headache, fever or other viral symptoms and followed by psychiatric and behavioural problems (including anxiety, unusual behaviour, delusions and insomnia) then progressing to impairment of consciousness, seizures, dyskinesias, choreoathetoid movements, autonomic instability and breathing problems (Armangue 2012). Presenting symptoms in younger children reflect disruption in the limbic system, with behavioural changes (new-onset temper tantrums, agitation, aggression and changes in mood or personality), changes in speech (reduced, mutism, echolalia, perseveration), seizures and movement disorders.

In adults, 56\% of women with anti-NMDA receptor encephalitis have an underlying ovarian teratoma. The incidence of teratoma in females

\begin{tabular}{|c|c|}
\hline Prodromal phase & \\
\hline Fever, headache, virus-like symptoms (ofter & unnoticed) \\
\hline Psychiatric and behavioural problems & \\
\hline $\begin{array}{l}\text { Young children } \\
\text { - New-onset temper tantrums } \\
\text { - Agitation } \\
\text { - Aggression } \\
\text { - Changes in mood or personality } \\
\text { - Changes in speech, e.g. reduced speech, } \\
\text { mutism, echolalia, perseveration } \\
\text { - Insomnia (occasionally hypersomnia) }\end{array}$ & $\begin{array}{l}\text { Teenagers and adults } \\
\text { - Anxiety } \\
\text { - Bizarre behaviours } \\
\text { - Paranoid thoughts } \\
\text { - Insomnia (occasionally hypersomnia) }\end{array}$ \\
\hline Later features & \\
\hline $\begin{array}{l}\text { - Seizures } \\
\text { - Decreased levels of consciousness } \\
\text { - Movement disorders, e.g. dyskinesias, cl } \\
\text { - Autonomic dysfunction: } \\
\text { - urinary incontinence } \\
\text { - tachycardia } \\
\text { - hypertension } \\
\text { - hyperthermia } \\
\text { - cardiac dysrhythmia (less frequent in } \\
\text { - Hypoventilation (more common in adults }\end{array}$ & $\begin{array}{l}\text { reoathetoid movements, postures } \\
\text { ildren) }\end{array}$ \\
\hline $\begin{array}{l}\text { Within } 1 \text { month of disease onset, whatever } \\
\text { develop symptoms from at least three of th } \\
\text { - psychiatric features } \\
\text { - memory disturbance } \\
\text { - speech disorder } \\
\text { - seizures } \\
\text { - dyskinesias } \\
\text { - decreased level of consciousness } \\
\text { - autonomic instability } \\
\text { - hypoventilation }\end{array}$ & $\begin{array}{l}\text { 7e presentation, most patients will } \\
\text { following groups: }\end{array}$ \\
\hline
\end{tabular}

Stages of presentation of anti- $N$-methyl-D-aspartate (anti-NMDA) receptor encephalitis (after Armangue 2012). 
with anti-NMDA receptor encephalitis decreases to $31 \%$ in those younger than 18 years and $9 \%$ in children under 14 years of age (Florance 2009). The treatment of anti-NMDA receptor encephalitis with immunotherapy (first line: corticosteroids, intravenous immunoglobulins and, possibly, plasmapheresis; second line: rituximab, cyclophosphamide), together with prompt removal if a tumour is found, can result in good neurological outcomes at 24 months. Less severe symptoms, prompt treatment and use of second-line immunotherapies if first-line therapies failed were predictors of better outcome in an observational cohort study (Titulaer 2013).

\section{Rasmussen encephalitis}

Rasmussen encephalitis is a rare progressive disease affecting one cerebral hemisphere. Study of the disease's aetiology has been difficult because of diagnostic delay - current hypotheses include an inflammatory immune response to an antigen, either foreign (e.g. an infectious agent) or autoimmune. The clinical course of the illness includes a prodromal stage with infrequent seizures, then presents with frequent seizures of differing types in the same patient, including unilateral epilepsia partialis continua (continuous focal jerking of a body part), cognitive decline and emergence of a hemiplegia that fluctuates initially before becoming permanent, accompanied by a progressive loss of brain volume in one hemisphere (Varadkar 2014). Rasmussen encephalitis may be treated by hemispherectomy.

\section{Epilepsy}

The psychiatric complications of epilepsy are well recognised, although clinically often underdiagnosed and undertreated (Verrotti 2014). It is less well known that some forms of epilepsy have an autoimmune basis. For example, a specific type, faciobrachial dystonia, described in 2008, can precede the onset of limbic encephalitis. Typically, seizures are brief (a few seconds), affect one side of the face and the ipsilateral arm and are frequent (happening as many as 50 times per day). The unusual presentation of the seizures, especially in the context of behavioural and memory disturbance, can result in their mislabelling as being non-organic in origin. Faciobrachial dystonia does not usually respond to anti-epileptic medication. Early recognition and immunotherapy may reduce the degree of post-encephalitic memory and cognitive impairments (Irani 2013a). A large Finnish register study showed that children with an autoimmune disease had a fivefold increase in risk of epilepsy (Ong 2014).

\section{Demyelinating disorders}

Acute disseminated encephalomyelitis (ADEM) and multiple sclerosis are two autoimmune neurological demyelinating disorders that can occur in childhood. In comparison with multiple sclerosis, a life-long relapsing, degenerative condition with $2-5 \%$ of cases beginning in childhood, ADEM is more common in children under 10 years old (Eyre 2014). Multiple sclerosis is not diagnosed until at least two episodes have occurred; until then, the term clinically isolated syndrome (CIS) is used. Both ADEM and CIS can present with neurological symptoms, but ADEM also includes symptoms of encephalitis. Multiple sclerosis can have a significant impact on the functioning and quality of life of young patients: cognitive dysfunction affects about a third of children and adolescents, and more than half experience a psychiatric disorder (Chou 2014). ADEM has a relatively favourable outcome, with the majority of children having a single-episode monophasic form that resolves within weeks. Follow-up studies of children who have recovered from an episode of ADEM have shown longerterm effects on behaviour and cognition, although a study with longer follow-up has given hope that these effects improve over time (Suppiej 2014).

\section{Opsoclonus-myoclonus syndrome}

Opsoclonus-myoclonus or 'dancing eye' syndrome is a rare neurological condition in young children, and an autoimmune pathogenesis is hypothesised. Once thought to be solely a paraneoplastic condition, there is an association with neuroblastoma in about half of childhood cases. In addition to the jerky eye and limb movements, children can experience irritability and sleep disturbance (Sahu 2011). The possible impact on behaviour of high-dose steroid treatments in early childhood, together with the potential significance of behavioural symptoms as markers of disease progress, can lead to difficult and complex decisionmaking in the care of such unusual children.

\section{Aicardi-Goutières syndrome}

Aicardi-Goutières syndrome is a rare autosomal autoimmune encephalopathy that most often presents in the first year of life following normal pregnancy and seemingly normal initial development. Symptoms include irritability, sleeping and feeding problems and unexplained fever, followed by psychomotor delay, loss of previously acquired skills, neurological impairment and slower head growth (Orcesi 2009). Neonatal presentation can mimic a congenital infection, with neurological impairment, microcephaly, intracranial calcification, hepatosplenomegaly 
and self-resolving haematological abnormalities. Raised interferon-alpha (IFN- $\alpha$ ) levels, triggered by an inappropriate innate immune response, are responsible for the main features of the disease.

\section{Recognition}

An important role of the child psychiatrist is to gather a full history from children and their families, including developmental history, family history, psychiatric history and responses to therapies. Given the wide-ranging associated psychopathologies of autoimmune disorder, it is important to consider the full spectrum of psychiatric and behavioural symptoms while taking the history and examining mental state. The fundamental psychiatric skills of taking a careful history and mental state examination can aid diagnosis and prompt timely collaboration with paediatric colleagues. The following particular features in a child's history should arouse a degree of suspicion for an autoimmune process.

\section{Personal or family risk of autoimmune disorders}

It is recognised that there is a high degree of comorbidity among autoimmune disorders. When a child has an autoimmune disorder there is often a family history of either the same or another autoimmune disorder. Although further large population studies are required to investigate the patterns of individual and family co-occurrence, the concept of an autoimmune diathesis is widely accepted (Cooper 2009). A child with Down syndrome should be seen as at high risk by virtue of their genotype. Clinicians should maintain an up-to-date understanding of the developing field of genetic risk in autoimmune conditions.

\section{Recent and current physical illness}

A review of systems should be carried out, including constitutional symptoms that might indicate systemic disease, for example rash, fever and weight loss. Enquire about any recent contact with infectious diseases, foreign travel and immunisation history.

\section{Disease features suggestive of an organic aetiology}

If a disorder is of sudden, severe onset, if there are impairments of consciousness or memory, or loss of previously acquired developmental milestones, there should be a high degree of suspicion of an organic process.

\section{Neurological symptoms, abnormal movements and seizures}

In a recent study of children with autoimmune encephalopathies (anti-NMDA receptor encephalitis, autoimmune basal ganglia encephalitis or Sydenham's chorea), three neurologists assessed videos of the children's movements (Mohammad 2014). They found that: chorea and dystonia were experienced by children across all three diagnoses; children with anti-NMDA receptor encephalitis were more likely to have stereotypies and perseveration; children with autoimmune basal ganglia encephalitis were most likely to have akinesia and tremor; and all of the children with Sydenham's chorea had choreiform movements.

General physical and neurological examination can provide clues to a systemic illness - skin changes, growth disturbance/weight loss and gross neurological abnormalities may be readily observable in young people presenting to child and adolescent mental health services (CAMHS). However, more subtle signs may require examination by a specialist paediatric neurologist. As signs may be transient, video-recording of children undertaken by family or in the clinic can inform discussion with colleagues in neurology.

\section{Investigation}

A decision regarding the most appropriate investigations for each child will reflect the differential diagnoses based on findings from the history and clinical examination. The PANS Collaborative Consortium advocates a thoughtful approach to investigation in which a work-up for autoimmune encephalitis, systemic autoimmune disease and other inflammatory diseases should be carried out only in the presence of relevant symptoms (Box 5). This is because of the high frequency of falsepositive antibody tests, which cause avoidable distress to families already coping with severely ill children (Chang 2015). Early liaison with paediatric and neurology colleagues may help

\section{BOX 5 Psychiatric symptoms prompting further investigation}

The Pediatric Acute-onset Neuropsychiatric Syndrome (PANS) Consortium Guidance recommends that the following symptoms should prompt consideration of an autoimmune encephalitis work-up:

- delirium, psychosis and/or diffuse encephalopathy

- pervasive cognitive decline

- persistent memory impairment

- pervasive behavioural deterioration

- seizure

- movement abnormality not consistent with tics

(Chang 2015) 
guide investigation, minimising the discomfort of repeated tests for children. Table 1 summarises some possible investigations and their indications, together with points to discuss with paediatricians: the strategy chosen depends on the presentation.

\section{Treatments}

Suspicion for and prompt recognition of neuropsychiatric autoimmune disorders in children could offer a broader range of therapeutic options than the traditional psychiatric approaches. A dramatic example is given in the case study of a 29 -yearold man with treatable organic suicidal behaviour resulting from NMDA-receptor encephalitis (Irani 2013b). Careful psychiatric assessment will contribute to treatment decisions and may help to monitor treatment response. Specific treatments for autoimmune disorders include corticosteroids, plasma exchange, intravenous immunoglobulins or other immunotherapeutic drugs, such as cyclophosphamide and rituximab (Twit 2014). These are outside the toolkit of most child and adolescent psychiatrists and both assessment and management should be considered in liaison with specialised paediatric neurologists and/or rheumatologists.
Immunotherapy can have significant psychiatric sequelae. Two cases of psychosis in adolescents with SLE - one as a result of primary neuropsychiatric SLE and the other due to steroid therapy - illustrate the paradox that pharmacotherapy may be a treatment or a cause (Alpert 2014). The authors highlighted the role of the clinical history (e.g. exposure to steroids) and biomarkers (e.g. autoantibodies: antiribosomal P, antineuronal or antiphospholipid) in differentiating neuropsychiatric SLE from other presentations (such as thrombotic thrombocytopenic purpura, vasculitis or steroid-induced psychosis) and in guiding the management.

As with all clinical decisions, the risk-benefit ratio should be considered. The familiar evidencebased treatments in child and adolescent psychiatric practice must not be underestimated, particularly for children who are not acutely or life-threateningly unwell. Psychoeducation, cognitive-behavioural therapy, family/systems interventions and psychotropic medication may all play an important part in the holistic approach to treatment. Neuropsychological assessment and support for learning in liaison with school and

Laboratory investigations and imaging in autoimmune psychiatric presentations

\begin{tabular}{|c|c|c|}
\hline Investigation & Example indication/rationale & Example results \\
\hline Blood: differential and full blood counts & $\begin{array}{l}\text { Anaemia - as a result of inflammatory conditions or a } \\
\text { nutritional anaemia }\end{array}$ & $\begin{array}{l}\text { Persistent thrombocytopaenia/leucopaenia may be a } \\
\text { sign of systemic autoimmune diseases such SLE }\end{array}$ \\
\hline Nutrition screen & $\begin{array}{l}\text { Children with autism who restrict their diet; possible } \\
\text { malabsorption (e.g. suspected coeliac disease) }\end{array}$ & $\begin{array}{l}\text { Low vitamin } B_{12} \text { levels could suggest } B_{12} \\
\text { encephalopathy }\end{array}$ \\
\hline Endocrine screen & $\begin{array}{l}\text { Individuals with Down syndrome and others with } \\
\text { symptoms suggestive of hormonal disruption }\end{array}$ & $\begin{array}{l}\text { Elevated TSH and low levels of thyroid hormones } \\
\left(T_{3} \text { and } T_{4}\right) \text { in Hashimoto's disease }\end{array}$ \\
\hline $\begin{array}{l}\text { Erythrocyte sedimentation rate (ESR) } \\
\text { C-reactive protein (CRP) }\end{array}$ & Inflammatory markers & Elevated in systemic inflammatory processes \\
\hline Computed tomography (CT) & Exclusion of other pathologies & $\begin{array}{l}\text { Calcification may be suggestive and cerebrovascular } \\
\text { changes may present atypically }\end{array}$ \\
\hline Magnetic resonance imaging (MRI) & $\begin{array}{l}\text { May require general anaesthetic and is not necessarily } \\
\text { diagnostic in acute cases }\end{array}$ & $\begin{array}{l}\text { Whole-body MRI may be indicated when } \\
\text { paraneoplastic process is suspected }\end{array}$ \\
\hline Electroencephalogram (EEG) & May be diagnostic in encephalopathy & $\begin{array}{l}\text { Slowing of the normal background frequency is a } \\
\text { classic finding in encephalopathy } \\
\text { 'Extreme delta brush' is a pattern associated with } \\
\text { NMDA receptor encephalitis (Sethi 2014) } \\
\text { Increased spike and wave may be associated with } \\
\text { emerging seizure disorder }\end{array}$ \\
\hline Cerebrospinal fluid (CSF) & $\begin{array}{l}\text { Invasive but may be diagnostic of a range of } \\
\text { inflammatory processes, including infection }\end{array}$ & $\begin{array}{l}\text { Cells and culture may be diagnostic } \\
\text { Oligoclonal bands may be an indication for more } \\
\text { specific testing }\end{array}$ \\
\hline $\begin{array}{l}\text { NMDA receptor antibodies } \\
\text { Voltage-gated potassium channel antibodies } \\
\text { Thyroid autoantibodies, etc. }\end{array}$ & $\begin{array}{l}\text { As new forms of autoimmune disorder are recognised, } \\
\text { there is increasing specificity in tests available (see } \\
\text { Chang 2015) }\end{array}$ & $\begin{array}{l}\text { Anti-NMDA receptor antibodies may be found in acute } \\
\text { autoimmune psychotic presentation, but their absence } \\
\text { does not rule out another autoimmune mechanism and } \\
\text { further discussion is indicated } \\
\text { A range of antibodies might be sought in discussion } \\
\text { with expert colleagues } \\
\text { Elevated thyroid antibodies are diagnostic in } \\
\text { Hashimoto's disease }\end{array}$ \\
\hline
\end{tabular}


educational psychology may also be important as there may be long-term impact on cognitive functioning, attention and school performance in a child with no previous difficulties. Psychotropic medication may be considered as indicated, but caution is required when factors such as autonomic dysregulation are complications of an autoimmune presentation. Where a child presents as acutely unwell, monitoring of respiratory or cardiac side-effects of psychotropics may be best managed by paediatric staff in liaison with the psychiatric team.

\section{Conclusions}

Recognition of the co-occurrence of autoimmune syndromes has led to greater opportunities for the study of potential mechanisms and treatments than the 'organ-specific' single-specialty approach to individual disorders. This highlights the need for cross-specialty working and, where there are psychiatric symptoms, the child psychiatry team is an essential partner. The specific example of Down syndrome exemplifies the growing need for a multidisciplinary approach. The possible role of the paediatric team in screening children with a known autoimmune disease for psychopathology is outwith the scope of this article, but is nonetheless an important consideration in local service design. The liaison psychiatry perspective gives consideration to the interplay between the biological, psychological, social and family factors affecting a young person. Chang et al (2015) describe, in the case of PANS, symptoms such as prominent behavioural regression and resultant social disruptions that may give the appearance of a psychosocial rather than biological aetiology, especially in a child with a history of disruptive behaviour. Psychiatric symptoms related to autoimmune conditions may present in any setting and the developing literature should be held in mind in all CAMHS assessments. A careful history and physical and mental state examination are integral to the assessment, treatment planning and follow-up for children and young people with autoimmune disorders and psychiatric symptoms.

\section{References}

Alpert 0, Marwaha R, Huang H (2014) Psychosis in children with systemic lupus erythematosus: the role of steroids as both treatment and cause. General Hospital Psychiatry, 36: e541-2.

American College of Rheumatology (1999) The American College of Rheumatology nomenclature and case definitions for neuropsychiatric lupus syndromes. Arthritis \& Rheumatism, 42: 599-608.

Armangue T, Petit-Pedrol M, Dalmau J (2012) Autoimmune encephalitis in children. Journal of Child Neurology, 27: 1460-9.

Baker GH, Brewerton DA (1981) Rheumatoid arthritis: a psychiatric assessment. BMJ (Clinical Research Edition), 282: 2014.
Benros ME, Waltoft BL, Nordentoft M, et al (2013) Autoimmune diseases and severe infections as risk factors for mood disorders: a nationwide study. JAMA Psychiatry, 70: 812-20.

Benros ME, Eaton WW, Mortensen PB (2014) The epidemiologic evidence linking autoimmune diseases and psychosis. Biological Psychiatry, 75 : 300-6.

Cameron FJ, Northam EA (2012) Screening for psychological disorders in youth with Type 1 diabetes: who, when, what and how? Diabetes Management, 2: 513-20.

Cameron FJ, Scratch SE, Nadebaum C, et al (2014) Neurological consequences of diabetic ketoacidosis at initial presentation of type 1 diabetes in a prospective cohort study of children. Diabetes Care, 37 : 1554-62.

Chan DO (2000) Neurologic, ophthalmic, and neuropsychiatric manifestations of pediatric systemic lupus erythematosus. Optometry \& Vision Science, 77: 388-94.

Chang K, Frankovich J, Cooperstock M, et al (2015) Clinical evaluation of youth with Pediatric Acute Onset Neuropsychiatric Syndrome (PANS): recommendations from the 2013 PANS Consensus Conference. Journal of Child and Adolescent Psychopharmacology, 25: 3-13.

Chistiakov D (2007) Down syndrome and coexistent autoimmune diseases. Journal of Applied Biomedicine, 5: 71-6.

Chou IJ, Whitehouse WP, Wang HS, et al (2014) Diagnostic modalities in multiple sclerosis: perspectives in children. Biomedical Journal, 37: 50-9.

Cooper GS, Bynum MLK, Somers EC (2009) Recent insights in the epidemiology of autoimmune diseases: improved prevalence estimates and understanding of clustering of diseases. Journal of Autoimmunity, 33: 197-207.

Dalmau J, Gleichman AJ, Hughes EG, et al (2008) Anti-NMDA-receptor encephalitis: case series and analysis of the effects of antibodies. Lancet Neurology, 7: 1091-8.

Emmer BJ, van der Bijl AE, Huizinga TWJ, et al (2009) Brain involvement in rheumatoid arthritis: a magnetic resonance spectroscopy study. Arthritis \& Rheumatism, 60: 3190-5.

Essex MJ, Shirtcliff EA, Burk LR, et al (2011) Influence of early life stress on later hypothalamic-pituitary-adrenal axis functioning and its covariation with mental health symptoms: a study of the allostatic process from childhood into adolescence. Developmental Psychopathology, 23 : 1039-58.

Eyre M, Absoud M, Wassmer E (2014) Childhood CNS inflammatory demyelinating diseases. Paediatrics and Child Health, 24: 439-46.

Florance NR, Davis RL, Lam C, et al (2009) Anti-N-methyl-D-aspartate receptor (NMDAR) encephalitis in children and adolescents. Annals of Neurology, 66: 11-8.

Irani SR, Stagg CJ, Schott JM, et al (2013a) Faciobrachial dystonic seizures: the influence of immunotherapy on seizure control and prevention of cognitive impairment in a broadening phenotype. Brain, 136: 3151-62.

Irani SR, Vincent A, Jacobson L, et al (2013b) Organic neuropsychiatry: a treatable cause of suicidal behaviour. Practical Neurology, 13: 44-8.

Laws P, Young H, Warren R (2014) Psoriasis and comorbidities. In Advances in Psoriasis: A Multisystemic Guide (eds JM Weinberg, M Lebwohl): 277-99. Springer.

Leckman JF, Vaccarino FM (2015) Editorial commentary: 'What does immunology have to do with brain development and neuropsychiatric disorders?'. Brain Research, 1617: 1-6.

Ludvigsson JF, Reichenberg A, Hultman CM, et al (2013) A nationwide study of the association between celiac disease and the risk of autistic spectrum disorders. JAMA Psychiatry, 70: 1224-30.

Mohammad SS, Fung VSC, Grattan-Smith P, et al (2014) Movement disorders in children with anti-NMDAR encephalitis and other autoimmune encephalopathies. Movement Disorders, 29: 1539-42.

Morris ZS, Wooding S, Grant J (2011) The answer is 17 years, what is the question: understanding time lags in translational research. Journal of the Royal Society of Medicine, 104: 510-20.

\section{MCO answers \\ $1 c \quad 2 d \quad 3 b \quad 4 d \quad 5 b$}


Murphy TK, Patel PD, McGuire JF, et al (2015) Characterization of the pediatric acute-onset neuropsychiatric syndrome phenotype. Journal of Child and Adolescent Psychopharmacology, 25: 14-25.

Ong MS, Kohane IS, Cai T, et al (2014) Population-level evidence for an autoimmune etiology of epilepsy. JAMA Neurology, 71: 569-74.

Orcesi S, La Piana R, Fazzi E (2009) Aicardi-Goutieres syndrome. British Medical Bulletin, 89: 183-201.

Sahu JK, Prasad K (2011) The opsoclonus-myoclonus syndrome. Practical Neurology, 11: 160-6.

Sari E, Karaoglu A, Yesilkaya E (2011) Hashimoto's thyroiditis in children and adolescents. In Autoimmune Disorders: Current Concepts and Advances from Bedside to Mechanistic Insights (ed F-P Huang): 27-40. InTech.

Sethi NK, Kim KW, Sethi PK (2014) EEG and PET changes in anti-Nmethyl-D-aspartic acid receptor encephalitis. American Journal of Psychiatry, 171: 889-90.

Suppiej A, Cainelli E, Casara G, et al (2014) Long-term neurocognitive outcome and quality of life in pediatric acute disseminated encephalomyelitis. Pediatric Neurology, 50: 363-7.
Swedo SE, Leckman JF, Rose NR (2012) From research subgroup to clinical syndrome: modifying the PANDAS criteria to describe PANS (pediatric acute-onset neuropsychiatric syndrome). Pediatrics \& Therapeutics, 2 . 113.

Titulaer MJ, McCracken L, Gabilondo I, et al (2013) Treatment and prognostic factors for long-term outcome in patients with anti-NMDA receptor encephalitis: an observational cohort study. Lancet Neurology, 12: $157-65$.

Tonutti E, Bizzaro N (2014) Diagnosis and classification of celiac disease and gluten sensitivity. Autoimmunity Reviews, 13: 472-6.

Twit M, Benseler SM (2014) Childhood inflammatory brain diseases: pathogenesis, diagnosis and therapy. Rheumatology, 53: 1359-68.

Varadkar S, Bien CG, Kruse CA, et al (2014) Rasmussen's encephalitis: clinical features, pathobiology, and treatment advances. Lancet Neurology, 13: 195-205.

Verrotti A, Carrozzino D, Milioni M, et al (2014) Epilepsy and its main psychiatric comorbidities in adults and children. Journal of the Neurological Sciences, 343: 23-9

Williams KA, Swedo SE (2015) Post-infectious autoimmune disorders: Sydenham's chorea, PANDAS and beyond. Brain Research, 1617: 144-54.

\section{MCQs}

Select the single best option for each question stem

1 Which of the following statements in relation to autoimmune presentations is untrue?

a careful exploration of the history can guide management of autoimmune presentations

b coeliac disease may present with irritability and fatigue

c PANDAS typically presents within 2 weeks of group A haemolytic streptococcus infection

d suicidality may be a presenting sign of autoimmune disorder

e there are well-recognised mechanisms for neuropsychiatric presentation of diabetes mellitus.

2 Which of the following examinatio ns and investigations is least likely to be useful when autoimmune disorder is suspected?

a careful examination of movement disorder presentations to clarify the diagnosis of autoimmune disease b mental state examination to discriminate likely cases of autoimmune encephalitis in children

c an EEG to help in deciding whether there are organic factors contributing to an acute psychiatric presentation

d an MRI brain scan in possible Sydenham's chorea

e lumbar puncture in atypical presentation of psychosis.

3 Which of the following is least likely to be useful in treatment of autoimmune neuropsychiatric disorders?

a cognitive-behavioural therapy for OCD in PANDAS

b plasmapheresis as a first-line treatment for PANDAS

c educational intervention after anti-NMDA receptor psychosis

d clonidine in tic disorders following Sydenham's chorea

e high-dose steroids in SLE psychosis.
4 What is the likely prevalence of neuropsychiatric symptoms in SLE?

a $0-5 \%$

b $5-15 \%$

c $15-25 \%$

d $45-55 \%$

e $75-80 \%$.

5 Which of the following autoimmune disorders may be a sign of underlying malignancy?

a SLE

b anti-NMDA receptor encephalitis

c Aicardi-Goutières syndrome

d coeliac disease

e Hashimoto's disease. 